\title{
El impulso de actividades innovadoras como apoyo a la docencia de asignaturas de base teórica. Enfocado a la docencia de las asignaturas pertenecientes al Departamento de Composición Arquitectónica \\ How innovative teaching activities can help theorical-based subjects. Focused on teaching subjects at the Architectural Composition Department
}

\author{
Ramón-Constantí, Amanda; Catalán-Tamarit, Francisco \\ Alumna de Grado en Fundamentos de la Arquitectura, ETSA Valencia, España, amracon@arq.upv.es
}

\begin{abstract}
Subjects at the Architectural Composition Department are distributed among the five years of the Architecture studies. These subjects are basically based on the study of History and cultural references, which has been studied traditionally by memory. In order to improve the teaching at these subjects and make the concept assimilation easier for pupils, teachers have been testing different methods on their classes to encourage the participation of students as well as achieve better grades. All these methods include the use of new technologies and complementary activities at the classroom as writing blogs, going on tours to local buildings treated at the master classes and the participation on activities at cultural architecture festivals from both teachers and students, who may later add to the regular teaching some of the topics seen at these fests as well as creating relationships with national and international Schools to see different approaches at the Composition subjects.

Keywords: Composition, motivation, flip teaching, innovation, history, teaching
\end{abstract}

\begin{abstract}
Resumen
Las asignaturas del Departamento de Composición Arquitectónica de la Escuela Técnica Superior de Arquitectura de Valencia, están distribuidas a lo largo de los 5 cursos de Grado. Estas asignaturas están basadas en el estudio de la historia y las referencias culturales que enriquecen la formación del futuro graduado y cuya docencia ha sido tradicionalmente evaluada a través de pruebas de nivel enfocadas a que el alumno aprenda de memoria. Con motivo de enriquecer la docencia de las asignaturas pertenecientes a este Departamento, los profesores han impulsado diferentes metodologías que permitan al alumnado una mayor implicación con estas materias, además de una mejor asimilación de conceptos y mejora de calificaciones. Estos métodos incluyen el uso de TIC y nuevas tecnologías, además de actividades complementarias en el aula que fomentan las competencias transversales del alumnado, todo ello con el objetivo de obtener una mejor docencia en las asignaturas del Departamento de Composición.
\end{abstract}

Palabras clave: Composición, motivación, docencia inversa, innovación, historia, docencia 


\section{Las asignaturas de base teórica en el Grado en Arquitectura}

El Grado en Fundamentos de la Arquitectura consta de 360 créditos, repartidos en 5 cursos con un programa académico que contiene los conocimientos básicos que debe tener el alumno para desarrollar el ejercicio profesional de la arquitectura una vez graduado. La del arquitecto es una profesión que requiere no sólo de amplios conocimientos técnicos y de base numérica a estudiar en su mayoría mediante casos prácticos, si no que también bebe de las ramas artísticas y más relacionadas con el diseño, donde queda patente la alta carga de créditos que poseen las asignaturas proyectuales en las que el alumno aprende a base de procesos pruebaerror y repetición de modelos. Estos modelos y referentes, tanto clásicos como modernos no suelen ser abordados en profundidad en las asignaturas más prácticas, aunque es absolutamente necesario su conocimiento y correcto manejo para que el alumno tenga una base cultural y arquitectónica desde la que partir.

\subsection{El Departamento de Composición Arquitectónica y sus asignaturas}

El Departamento de Composición Arquitectónica de la Escuela Técnica Superior de Arquitectura (ETSA) de Valencia es el órgano responsable de organizar y desarrollar la investigación y docencia propias del área de conocimiento de la Composición Arquitectónica, y cuenta en la actualidad con asignaturas repartidas a lo largo de los cinco cursos del Grado en Fundamentos de la Arquitectura, materias en los diferentes Máster impartidos en la Escuela así como optativas propias en otras Escuelas.

Las asignaturas pertenecientes a este Departamento tienen en su mayoría una importante carga teórica y son un área singular, de corte más humanística, pero que el futuro arquitecto debe dominar tanto como el cálculo o la construcción. Son aquéllas materias cargadas de obras, autores y referentes a lo largo de los siglos, y que se han evaluado tradicionalmente a través de pruebas de nivel y exámenes que exigen al alumno no sólo asistir a clase y producir unos apuntes de calidad, si no también dedicar horas a memorizar e interiorizar el contenido tratado en el aula. En un Grado con la mayor parte de sus asignaturas enfocadas a la práctica y la producción de material gráfico y proyectual, esto lleva a que muchos alumnos desconozcan cómo enfrentarse a estas materias a nivel de estudio y trabajo en clase.

\section{Los recursos tradicionales en la docencia de las asignaturas del Departamento}

Historia, corrientes filosóficas y artísticas, geografía, composición... Son algunos de los contenidos que tratan las asignaturas del Departamento de Composición, y que tradicionalmente han sido parte de la docencia en el aula mediante libros de texto, diapositivas, citas y libros de lectura obligatoria, entre otros. Si bien la metodología tradicional ha ido eriqueciéndose con la incursión de nuevas tecnologías en las aulas a lo largo de los años, todavía hoy en día no se aprovecha el cien por cien de los recursos disponibles.

La proyección audiovisual es una herramienta que ha ido sustituyendo poco a poco los libros de texto, utilizándola como soporte para la explicación del temario y la realización de debates y otras actividades mediante diapositivas proyectadas en el aula. Los trabajos monográficos, individuales o por equipos suelen ser tareas puntuables pero a realizar fuera del aula, impidiendo esto en muchas ocasiones la realización de actividades en clase que de ser realizadas por equipos con un mayor número de componentes, o incluso coordinando a todos los alumnos a participar en una sesión, podrían resultar más didácticas y enriquecedoras. 
También es necesario tener en cuenta los actuales mínimos, programaciones y competencias evaluables exigidos por parte de las Comisiones Académicas y organismos reguladores del Grado, que imposibilitan en muchas ocasiones valorar competencias relacionadas directamente con la docencia de muchas asignaturas, pero que no aparecen en los currículums académicos, como pueden ser las competencias transversales o el conocimiento e interés por parte del alumno de contenidos, referentes u obras que no aparecen en el temario. Esto lleva a que el sistema de evaluación y calificación sea sistemático en gran parte de las asignaturas del Departamento y del Grado en su totalidad mediante pruebas de nivel y exámenes, prácticas evaluables y en definitiva, evaluaciones poco flexibles teniendo en cuenta el amplio área de conocimiento abarcado.

\section{La motivación del alumno: el motor para impulsar actividades innovadoras}

El papel del docente es fundamental para obtener de sus alumnos el mayor y mejor rendimiento posibles dentro de su área de docencia. El docente es el/la encargada en todo momento no sólo de transmitir el conocimiento y cumplir los objetivos establecidos para el correcto desarrollo del curso, si no también de mantener el interés del alumno por el temario impartido. Esto lleva al siguiente nivel, donde entra en juego la motivación. Si el alumno no sólo muestra interés por la materia, si no que además las tareas y prácticas programadas lo motivan e implican en el desarrollo de la clase, el rendimiento mejorará y además, contribuirá a desarrollar nuevas actividades en el aula. En la mayoría de ocasiones, éstas resultan en prácticas y trabajos académicos innovadores, fuera de lo común en su ámbito y que destacan positivamente. Esto sienta precedente para futuros cursos, donde el docente puede repetir la experiencia con nuevos alumnos, fomentando nuevas actividades, enriqueciendo la docencia e implicando en mayor grado al alumnado en el temario impartido y las prácticas realizadas.

Una actividad innovadora es aquélla coherente con los criterios de calidad de la docencia, pero que además potencia el desarrollo de formas de actividad conjunta entre docente y alumnos, en la que es posible prestar ayuda educativa ajustada a los contenidos de parte del primero, y colabora a la autorregulación del aprendizaje del segundo (Mauri, 2007). Las metodologías innovadoras potencian la actividad conjunta de profesores y alumnos, favorecen el trabajo cooperativo como instrumento docente, y en casi todos los casos, utiliza las TIC como herramientas de ayuda para un mejor aprendizaje del alumno y entre alumnos.

Para que tanto las instituciones existentes como las que están aún dando sus primeros pasos puedan responder al desafío que significa innovar en la docencia, éstas deben revisar sus referentes actuales y promover experiencias innovadoras en el proceso enseñanza-aprendizaje (Salinas, 2004). Así, hacer énfasis en la docencia mediante estrategias didácticas innovadoras que incluyan cambios en las herramientas en el aula o el sistema tradicional de docencia, contribuyen en los procesos de innovación docente y a reforzar la confianza en las capacidades creativas de cada alumno.

Como ejemplo reseñable, sería aquél alumno al que le es encomendada una práctica puntuable en un formato normalizado, como un panel o un trabajo monográfico encuadernado, pero que por iniciativa propia y a riesgo de obtener una calificación menor que la de sus compañeros (o incluso arriesgarse a no ser puntuado en el peor de los casos) decide obviar el formato establecido y adaptar la temática y contenido del trabajo a un videoclip, una canción, juego de mesa o conjunto de diapositivas, entre otros. Esto no sólo demuestra iniciativa, si no que también es un interesante ejercicio de creatividad y esfuerzo por parte del alumno en 
demostrar competencias provenientes de otros ámbitos, incluir en mayor medida el uso de las TIC y aprovechar al máximo los recursos disponibles en el aula, así como diferenciarse del resto de compañeros.

\section{Recursos innovadores y TIC actualmente aplicadas a la docencia en las asignaturas del Departamento}

Actualmente en la Escuela Técnica Superior de Arquitectura de Valencia destacan como precursoras de actividades docentes innovadoras prácticamente la totalidad de las asignaturas pertenecientes al Departamento de Composición. Éstas son un excelente referente para otras materias del Grado, no sólo por pertenecer a un Departamento presente a lo largo de los cinco cursos en la vida académica de cualquier estudiante de esta Escuela, si no también por ser pioneras en experimentación. Mediante diferentes actividades que se citarán a continuación, el alumno es capaz de aplicar las competencias transversales, además de hacer un ejercicio de creatividad y uso de la imaginación propio del Grado en Fundamentos de la Arquitectura.

-Cuaderno de práctica de campo: es la práctica evaluable con mayor peso en la asignatura cuatrimestral de Introducción a la Arquitectura en primer curso. Consiste en un cuaderno de formato manejable que los alumnos llevan consigo para, a modo de diario de viaje, anotar, dibujar, pegar fotografías, hacer collage, etc. De todo aquello que les llama la atención durante los primeros meses en la Escuela de Arquitectura. El cuaderno de práctica de campo debe documentar un par de actividades obligatorias todos los cursos, que varían de año en año, pero que básicamente consisten en actividades que llevan al alumno a explorar la ciudad y su entorno más inmediato, dibujando y fotografiando, a modo de "safari" para posteriormente comentar en el mismo cuaderno aquello que ha encontrado. Desde un edificio que le ha resultado singular, hasta algún detalle concreto de una puerta o ventana, pasando por alguna paleta de colores o aproximación a proceso constructivo, todo ello contribuye a que el alumno comience a asimilar la gran cantidad de conceptos nuevos que lo rodean.

-Blogs: es la actividad que actúa a modo de espina dorsal de las prácticas obligatorias y puntuables, además de algunas optativas de la asignatura de Historia del Arte impartida en segundo curso. Se encomienda a los alumnos dirigir un blog en grupos, donde publicarán las prácticas realizadas en clase, a modo de artículos, reseñas bibliográficas, comentarios acerca del temario impartido en el aula, etc.

Esta actividad resulta enriquecedora por diferentes motivos. Estos incluyen el trabajo en equipo en un formato informático poco habitual en el aula, la inmediatez de producción y publicación de contenidos (lo que provoca que el alumno no sólo publique las prácticas obligatorias, si no que dedique tiempo a investigar y compartir acerca de otras inquietudes), el hecho de que los compañeros y el público puedan visualizar y opinar del trabajo ajeno, además de en muchos casos, extenderse la actividad del blog una vez finalizada la asignatura. 


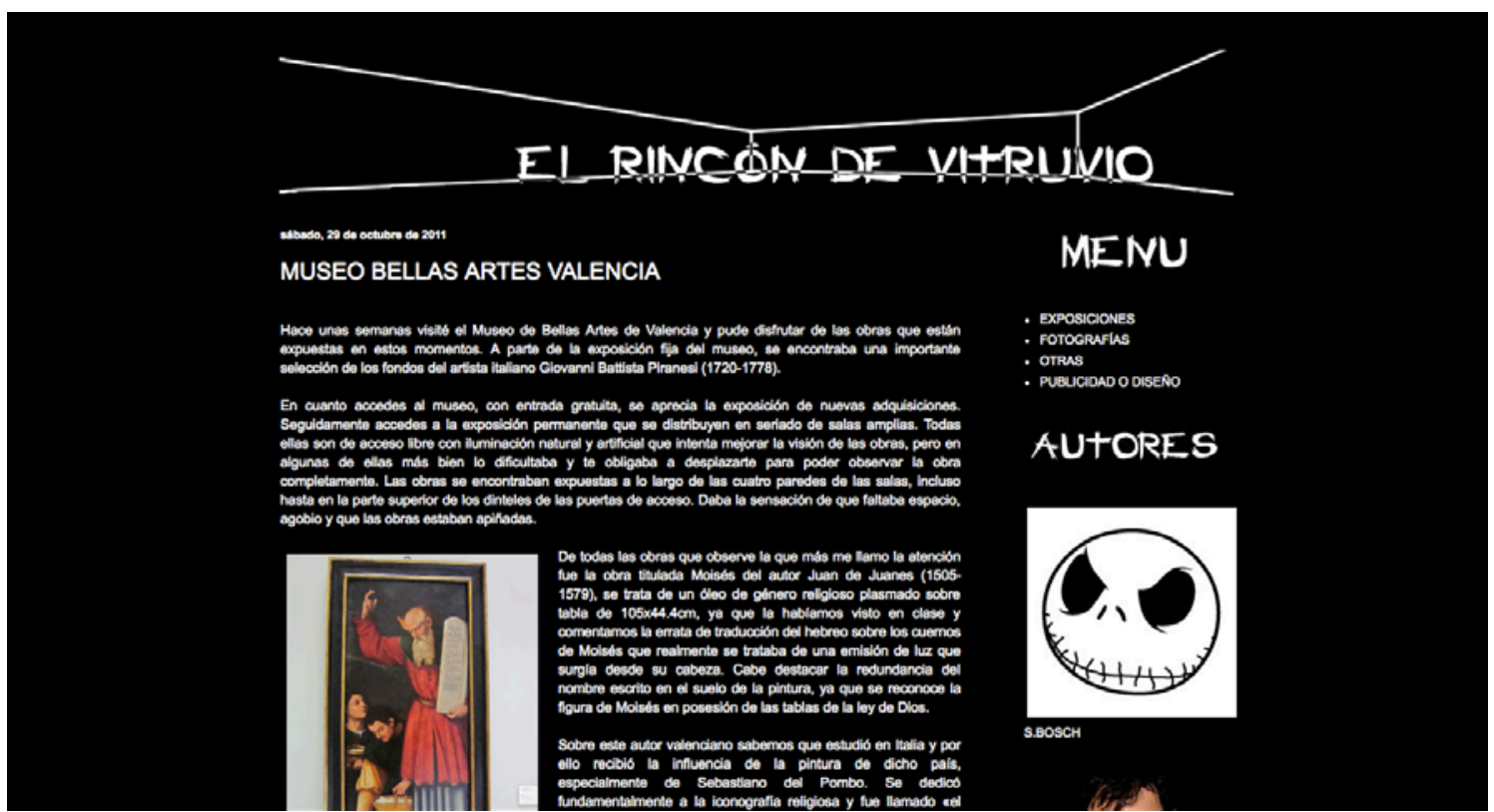

Fig. 1 Captura del blog “EL RINCÓN DE VITRUVIO", práctica de aula de un grupo de alumnos de la asignatura de Historia del Arte

-Material audiovisual: siendo el temario tratado en la mayoría de las asignaturas del Departamento de Composición Arquitectónica de un componente teórico, cuyo enfoque en el aula se realiza mediante diapositivas estáticas, resulta interesante incluir también material audiovisual en el aula. Éste pueden ser cortos, publicidad, música, etc. Relacionados directamente con el tema tratado, e incluso, animar a los alumnos a producirlos como prácticas evaluables. Como material en el aula, el material audiovisual resulta de gran utilidad como anécdota o hecho puntual para destacar contenidos, para facilitar la asimilación de conocimientos de una forma amena y sencilla.

Como práctica evaluable y de producción propia del alumno, este tipo de ejercicios exigen al alumno un mayor esfuerzo creativo y de relación de conceptos, así como ciertas capacidades técnicas como manejo básico de equipos de audio y sonido o programas especializados. No obstante, también es cierto que resultan trabajos mucho más ricos, con una mayor implicación de parte del alumnado en comparación a otros recursos, y que pueden conducir a correcciones y visionados grupales que contribuyan a compartir el proceso de ideación, realización y la crítica constructiva. 


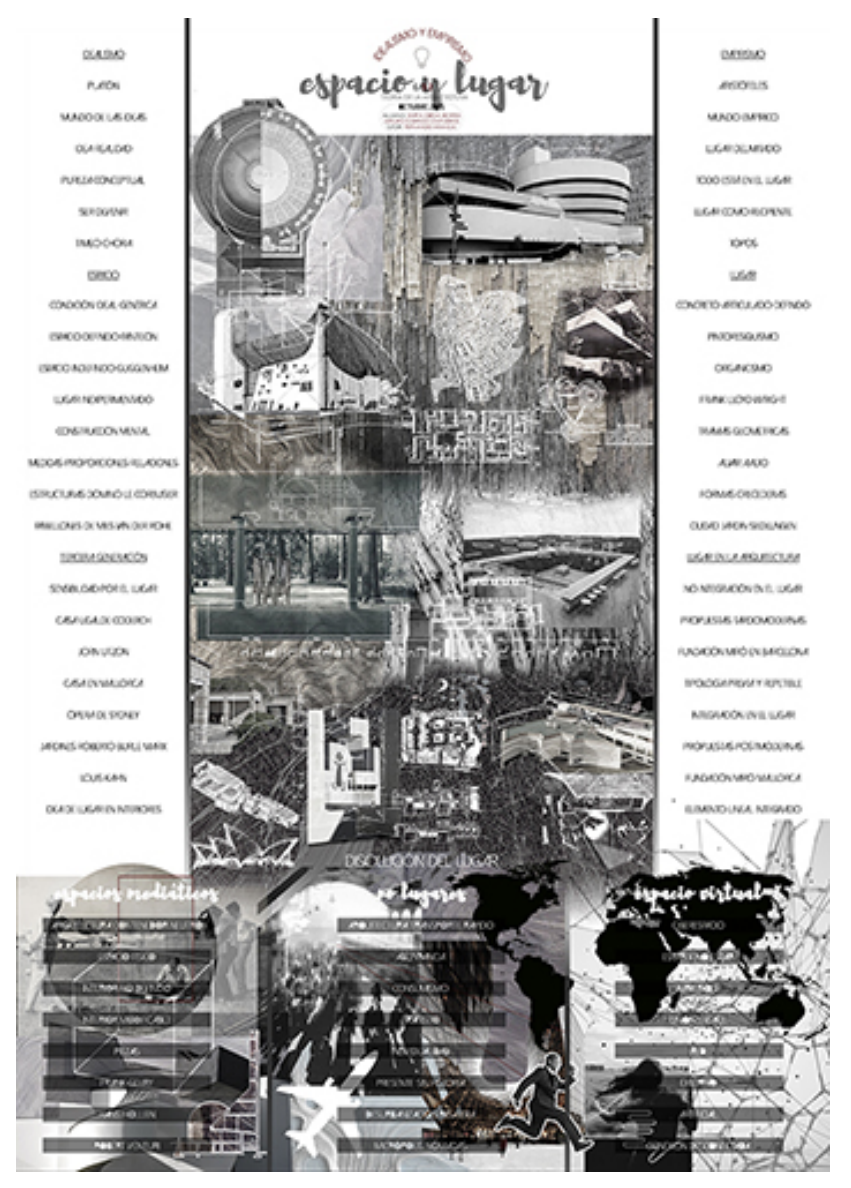

Fig. 2 Práctica puntuable en formato panel DIN-A1 de Sofía Úbeda y Arturo Garrido, para la asignatura de Teoría de la Arquitectura

-Visitas guiadas: las salidas y visitas a lugares singulares tratados en el aula, o que por su cercanía o contexto histórico resulten complementarios al temario son una actividad muy didáctica. Estas visitas acercan al alumno al espacio tratado en clase, pasando de mero espectador a ser partícipe de la arquitectura que le rodea, poniéndola en valor y asimilando de una forma más directa los conceptos e información previamente estudiados, y convirtiéndolos en una experiencia. El alumno no sólo se limita a mirar: puede tocar, recorrer el lugar, oler...

Además, es una excelente forma de acercar y dar a conocer la arquitectura y cultura locales, poniendo en valor edificios y lugares que pertenecen al día a día del alumno y son fácilmente identificables.

-Prácticas evaluables en formatos poco comunes: el DIN-A4 es un formato que resulta cómodo por ser manejable y ampliamente utilizado en el día a día. No obstante, éste resulta restrictivo a la hora de exponer gráficos, diseños y prácticas diseñadas acorde al ámbito tratado en un Grado de Fundamentos en la Arquitectura. Aunque es imprescindible un correcto uso del lenguaje, la gramática y saber demostrar los conocimientos adquiridos mediante la redacción de textos, también es importante poseer conocimientos de maquetación, tipografía, así como de storytelling y realización de infografías entre otros. Las asignaturas que pertenecen al Departamento de Composición Arquitectónica son un excelente marco para este tipo de prácticas y ejercicios. Más allá del trabajo monográfico, éstos tienen la capacidad de impulsar y valorar la creatividad y la innovación de parte del alumnado, convirtiendo sus prácticas y trabajos en elementos útiles fuera del aula una vez concluida la asignatura. 
Como resultado a estas prácticas, donde el alumno se ve implicado en una mayor medida y suele prestar más interés en su realización, pueden surgir formatos libres, o complementarios al trabajo monográfico como maquetas, juegos de mesa, paneles informativos, rompecabezas, dioramas...

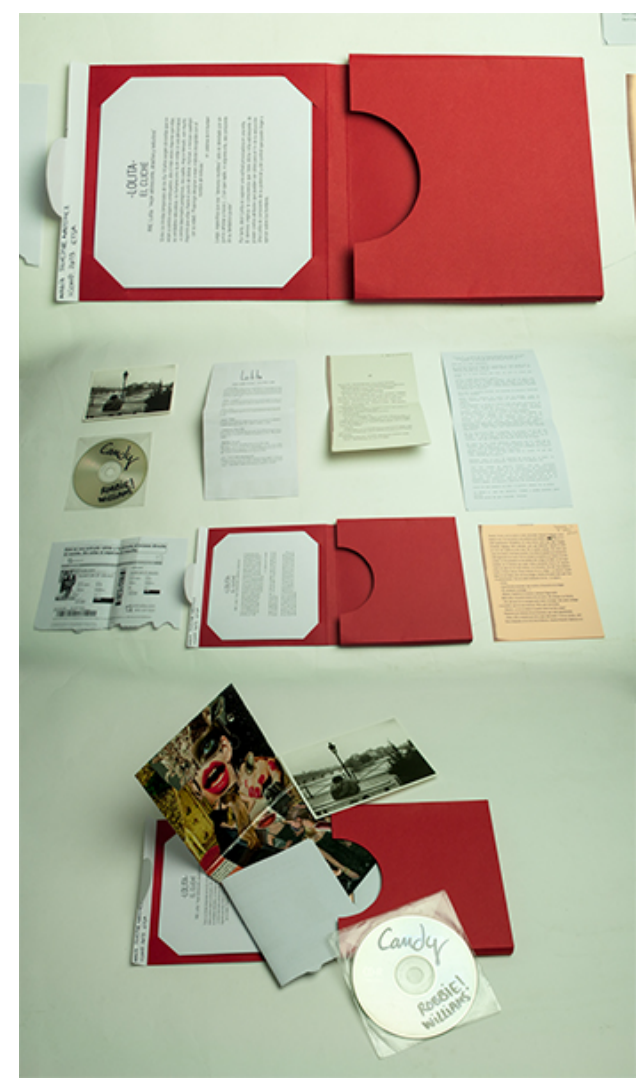

Fig. 3 Trabajo en formato libre de María Silvestre sobre el libro "Lolita", para la asignatura de Atmósferas

-Docencia inversa: o flip teaching, consiste en invertir el sistema de aprendizaje tradicional, siendo la teoría, diapositivas y clases magistrales contenido que el alumno visiona en sus horas de estudio en casa, mientras que las prácticas y exposiciones grupales se realizan en el aula. Es así como se obtiene un mayor rendimiento del alumno y un mayor aprovechamiento de las horas junto al docente, que es capaz de resolver dudas generales o puntuales de cara a todo el grupo, y prestar asistencia más fácilmente en las prácticas puntuables y proyectos finales.

-Fomento de actividades culturales extracurriculares: mediante la participación en actividades culturales fomentadas a través de la Escuela, tanto del docente como del alumno, se pueden lograr sinergias muy interesantes que conduzcan a debates, mesas redondas $u$ otras actividades similares en el aula. Como ejemplo, una mesa redonda con profesores de varios departamentos, entre ellos del Departamento de Composición, en el festival cultural enfocado a la arquitectura "ETSATOPIA" que tuvo lugar en la Escuela Técnica Superior de Arquitectura de Valencia el pasado curso 2015-2016. Esta actividad reunió a una gran cantidad de alumnos interesados en el debate llevado a cabo, y cuyo formato se llevó a clases posteriores creando debate acerca de diferentes aspectos del temario. 


\subsection{Discusión de encuesta al alumnado acerca de metodologías docentes}

Para obtener datos acerca de cómo afectan las actividades innovadoras a la docencia y si la percepción del alumno en cuanto a estas metodologías es positiva, se realizó una encuesta online. Ésta tenía dos partes diferenciadas pero con preguntas similares: una enfocada a aquéllos alumnos que sí que habían recibido docencia en cualquier asignatura mediante actividades innovadoras, y otra parte enfocada a aquéllos que no.

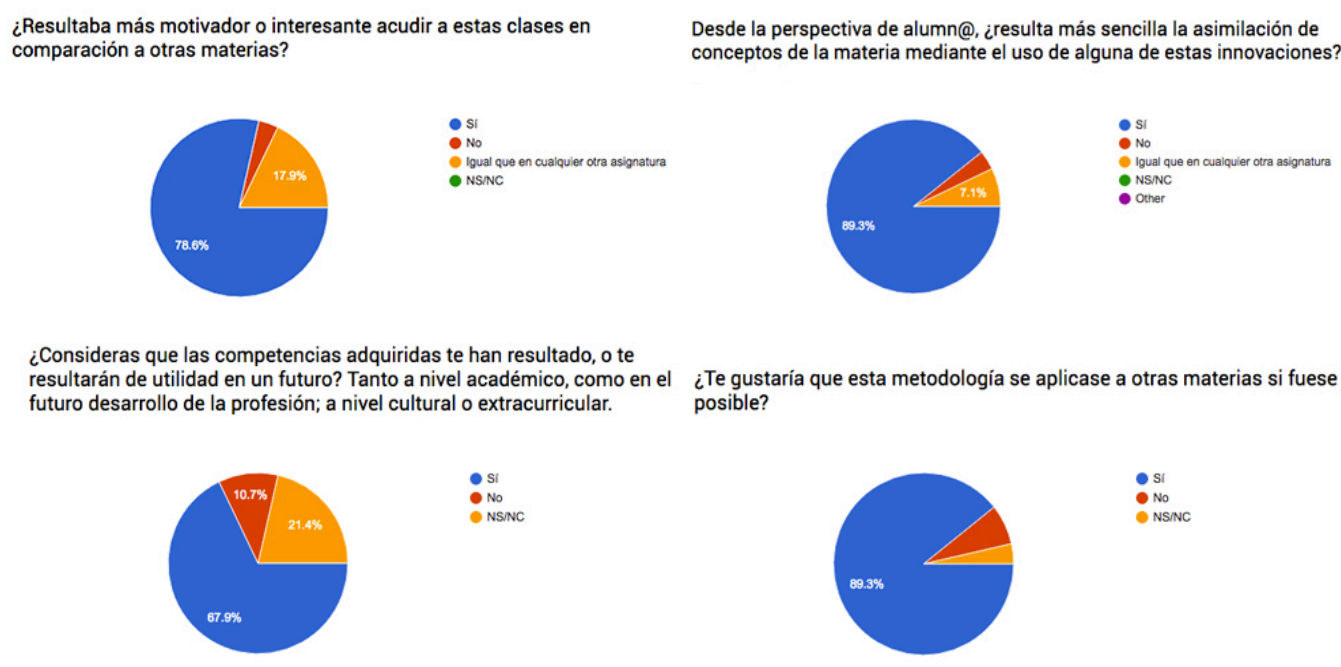

Fig. 4 y 5 Resultados de la encuesta a los alumnos que sí habían recibido docencia, en cualquiera de las asignaturas del Departamento de Composición Arquitectónica mediante actividades innovadoras

Por otra parte, las siguientes preguntas se realizaron a aquéllos alumnos que nunca habían recibido docencia mediante actividades innovadoras. Los resultados, aunque en apariencia similares entre aquéllos que no recibieron docencia mediante actividades innovadoras y los que sí, muestran que aquéllos alumnos que realizaron blogs, ejercicios mediante material audiovisual y similares, se sentían más motivados frente a otras asignaturas con una docencia con materiales tradicionales, e incluso les gustaría que este tipo de innovaciones se utilizaran para trabajar en otras asignaturas.

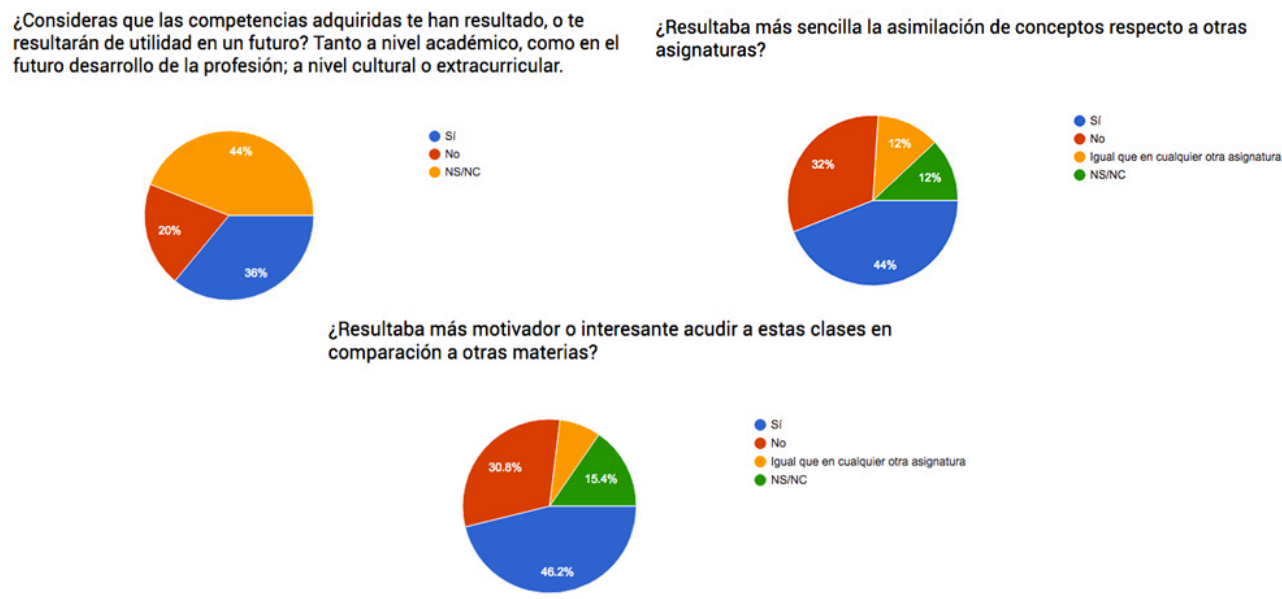

Fig. 6 Resultados de la encuesta a los alumnos que no recibieron docencia mediante actividades innovadoras 


\section{Conclusiones}

La creatividad es sin duda alguna, el motor de las actividades innovadoras en el aula, de parte tanto de alumnos como de docentes. Mediante los datos recogidos en la encuesta realizada a 40 alumnos, se deduce que el alumno que sólo ha recibido docencia mediante metodologías tradicionales no valora la posibilidad de que otro tipo de actividades sean factibles para mejorar la asimilación de conceptos y la motivación a la hora de estudiar una asignatura. Sin embargo, aquéllos alumnos que sí que han recibido alguna vez docencia mediante metodologías innovadoras y que potencian las TIC y la creatividad, aprecian los cambios e incluso adaptarían estos métodos docentes a otras materias para lograr un mejor rendimiento. Es también destacable cómo, mientras que los alumnos que recibieron docencia innovadora valoran positivamente las competencias que adquirieron o pudieron demostrar en el aula; el resto del alumnado no es capaz de ver el potencial que pueden tener las competencias adquiridas mediante la docencia más tradicional, respondiendo a la pregunta formulada con un "no sabe, no contesta".

En una época donde se continúa un enfoque educativo con métodos tradicionales de enseñanza en entornos que ya no son tan tradicionales, lograr la innovación en todas las áreas de conocimiento de un Grado universitario debería ser una tarea prioritaria desde las instituciones, y donde deben verse involucrados todos los factores. Ya no sólo hablando de prácticas y ejercicios puntuales a nivel aula o curso como los citados anteriormente, si no trasladar la innovación a todas las áreas de conocimiento y disciplinas posibles. La innovación en las aulas es un factor importante que actúa como catalizador de la creatividad, y ésta a su vez, un importante principio para entender la educación y actividades profesionales del futuro.

La creatividad es tan importante en la educación como la alfabetización, y por eso debemos tratarla con la misma importancia (Robinson, 2009). Hoy en día, en un mundo donde las TIC están presentes en cualquier ámbito y a toda hora, también el las formas de aprendizaje deberían variar tan vertiginosamente como lo hacen las tecnologías. La preparación de los futuros arquitectos y graduados universitarios debería asumir los cambios y responsabilidades que se necesitan en un mundo rápido y donde aquéllo que era nuevo hace dos días, ya no lo será dentro de dos meses.

\section{Agradecimientos}

A Victoria Bonet Solves, por las sugerencias e ideas para el desarrollo de este artículo.

A los/as alumnos que han permitido la captura y posterior inclusión en este artículo de sus trabajos o prácticas de aula innovadoras.

\section{Referencias}

BOSCH, S.; QUENTIN; AMEN; MONTI; PUIG, J.E.; GUICAR . El rincón de Vitruvio. <http://elrincondevitruvio.blogspot.com.es/ > [Consulta: 13 de julio de 2016]

MAURI, T. ; COLL, C ; ONRUBIA, J. (2007) "La evaluación de la calidad de los procesos de innovación docente universitaria. Una perspectiva constructivista" Red U. Revista de Docencia Universitaria. $N^{\circ} 1$ [Consulta: 13 de Agosto de 2016]. http://www.redu.um.es/Red_U/1/

ROBINSON, K. ; ARONICA, L. (2012). El elemento. [s.I.]: CONECTA. 
SALINAS, J. (2004). "Innovación docente y uso de las TIC en la enseñanza universitaria". Revista de Universidad y Sociedad del Conocimiento (RUSC). UOC. Vol. 1, n 1. [Consulta: 1 de agosto de 2016]. http://www.uoc.edu/rusc/dt/esp/salinas1104.pdf

TED, "The surprising habits of original thinkers | Adam Grant". Youtube < https://www.youtube.com/watch?v=fxbCHn6gE3U > [Consulta: 1 de agosto de 2016] 